\title{
Recommending esketamine? 4 factors to consider
}

Diana Samuel, MD, Joshua Berman, MD, PhD, and Ravi N. Shah, MD, MBA

$\mathrm{S}$ ince receiving FDA approval in March 2019, intranasal esketaminethe S-enantiomer of ketamine-has become a valuable treatment option for adults with treatment-resistant depression (TRD), owing to its limited adverse effects profile, rapid onset, and potential for significant improvement in depressive symptoms. In August 2020, the FDA expanded esketamine's indication to include treatment of depressive symptoms in adults with acute suicidal ideation or behavior, thus providing psychiatrists with an additional option for improving the care of their most seriously ill patients. In this article, we review 4 factors to consider before recommending esketamine.

1. Confirm that the patient's depression qualifies as treatment-resistant. A patient is considered to have TRD if they have longstanding depression that meets DSM-5 criteria for major depressive disorder, and have not adequately responded to at least 2 antidepressant trials of adequate dose and duration.

2. Confirm that the patient's suicidal ideation and/or suicidal behavior does not require acute hospitalization. The time between the onset of suicidal ideation and a suicide attempt typically is short, which highlights the need to intervene quickly in these patients. ${ }^{1}$ Being able to provide a treatment that works quickly and effectively may be lifesaving. However, to receive esketamine, patients must be enrolled in the Risk Evaluation Mitigation Strategy (REMS) patient registry through a certified treatment center, and prior authorization from insurance generally is required. These steps take time, so patients at high or imminent risk for suicide may initially require psychiatric hospitalization before they are able to begin esketamine treatment. Parsing out whether the suicidal ideation is chronic or acute can help clinicians assess current dangerousness and determine if esketamine treatment might be appropriate. If a patient with chronic suicidal ideation is stable for outpatient treatment with close monitoring, esketamine might provide an effective treatment option for treating both depression and suicidality. Esketamine's rapid effect may be an integral part of the treatment for a suicidal patient by bridging the gap caused by the delayed onset of action in typical antidepressants. ${ }^{2}$

3. Identify a local certified treatment center. Use the online database at www.spravato. com/find-a-center to locate a nearby certified esketamine treatment center. Choosing a center that you can collaborate with regularly is important to determine if the treatment is effective, to provide updates on the treatment course, and to consider tailoring of ongoing treatment.

4. Ensure the patient is also treated with an oral antidepressant. Esketamine should be

\section{Every issue of CURRENT PsychiatRY} has its 'Pearls'

\section{Yours could be found here.}

Read the 'Pearls' guidelines for manuscript submission at MDedge.com/ CurrentPsychiatry/page/pearls. Then, share with your peers a 'Pearl' of wisdom from your practice.
Dr. Samuel is Assistant Professor, Department of Psychiatry, Columbia University Irving Medical Center, New York, New York. Dr. Berman is Assistant Professor, Department of Psychiatry, Columbia University Irving Medical Center, New York, New York. Dr. Shah is Assistant Professor, Department of Psychiatry, Columbia University Irving Medical Center, New York, New York.

Disclosures

The authors report no financial relationships with any companies whose products are mentioned in this article, or with manufacturers of competing products.

doi: 10.12788/cp.0142

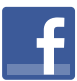

Discuss this article at www.facebook.com/ MDedgePsychiatry $\mathbf{0}$ 
administered in conjunction with an oral antidepressant. As such, patients must be willing and able to tolerate treatment with a medication that can be construed as an antidepressant while undergoing esketamine treatment. A long-term maintenance trial found that patients with TRD who experienced remission or response after esketamine treatment had a delayed relapse of symptoms when they continued esketamine in addition to an oral antidepressant. ${ }^{3}$

Considering its rapid onset of action and low adverse effects profile with manageable tolerability, esketamine adjunctive to an oral antidepressant is a reasonable option to consider for patients with TRD, including those with suicidality.

\section{References}

1. Deisenhammer EA, Ing CM, Strauss R, et al. The duration of the suicidal process: how much time is left for intervention between consideration and accomplishment of a suicide attempt? J Clin Psychiatry. 2009;70(1):19-24.

2. Canuso CM, Singh JB, Fedgchin M, et al. Efficacy and safety of intranasal esketamine for the rapid reduction of symptoms of depression and suicidality in patients at imminent risk for suicide: results of a double-blind, randomized, placebo-controlled study. Am J Psychiatry. 2018;175(7):620-630.

3. Daly EJ, Trivedi MH, Janik A, et al. Efficacy of esketamine nasal spray plus oral antidepressant treatment for relapse prevention in patients with treatment-resistant depression. JAMA Psychiatry. 2019;76(9):893-903. 